\title{
VALIUM ENDOVENOSO NO TRATAMENTO DO ESTADO DE MAL EPILÉPTICO
}

\author{
DécIo MEga * \\ MiCHeL P. LISON*
}

A recente obtenção dos derivados benzodiazepinicos veio abrir novas perspectivas na terapêtica da epilepsia. Embora a ação favorável dessas drogas ainda não tenha sido estabelecida em definitivo para os diferentes tipos de crises, seu uso parece ser particularmente útil no contrôle do "Pequeno Mal" 2, 3, 5, 7, dos espasmos em flexão 4, 6, 9, 11" e dos "estados dc mal epiléptico $2,8,11,12,13$.

No presente trabalho relatamos nossa experiência com o uso do Valium (diazepam) por via endovenosa (E.V.) em pacientes em "estado de mal epiléptico". Êste têrmo é aqui empregado em sentido mais amplo, englobando não apenas pacientes em "status epilepticus" - definido por Gastaut e col. ${ }^{1}$ - mas também aqueles com recuperação da consciência, nos periodos intercríticos ("serial epilepsy") 8 .

\section{MATERIAL E METODO}

Nosso material ccnsta de 25 pacientes admitidos nos últimos 8 meses, que apresentavam crises generalizadas ou lateralizadas, de freqüência nāo inferior a duas por hora, com ou sein recuperação da consciência, nos periodos intercriticos. Em 5 pacientes constatou-se afecção neurológica aguda; nos restantes, a provável ctiologia estava relacionada a lesão cerebral crônica, não determinada em 6 pacientes (Tabela $\mathbf{1}$ ).

O Valium foi administrado por via E.V., lentamente (2-3 $\mathrm{mg} / \mathrm{minuto})$, na dose média de 0,3 a $0,4 \mathrm{mg} / \mathrm{kg}$, para pacientes com pêso inferior a $25 \mathrm{~kg}$; a partir desta cifra, na dosagem única de $10 \mathrm{mg}$. Quando necessária, uma segunda dose era injetada, decorridos pelo menos 30 minutos, por via E.V. ou através de infusão E.V. pelo sistema gôta a gôta.

Considerou-se ótimo resultado a cessação imediata das crises, por espaço não inferior a 6 horas, após a primeira ou segunda dose; como regular a diminuição em sua frequiência; como mau, a inalterabilidade do número de crises, mesmo após aplicação de uma segunda dose.

Departamento de Neurologia (Prof. J. Armbrust-Figueiredo) da Faculdade de Medicina de Ribeirão Prêto da Universidade de São Paulo: "Assistentes.

Nota dos autores - Agradecemos a Produtos Roche Químicos e Farmacêuti$\cos$ S.A. pelo fornecimento do Valium. 


\begin{tabular}{|c|c|c|c|c|c|}
\hline Caso & Idade & $\begin{array}{l}\text { Tipos } \\
\text { de crises }\end{array}$ & Etiologias prováveis & $\begin{array}{l}\text { Doses } \\
\text { admi- } \\
\text { tradas }\end{array}$ & $\begin{array}{l}\text { Resul- } \\
\text { tados }\end{array}$ \\
\hline 1 & 36 anos & $\begin{array}{l}\text { Generalizada tô- } \\
\text { nico-clônica }\end{array}$ & - & 1 & ótimo \\
\hline 2 & 65 anos & Hemiclônica & Arteriosclerose & 1 & ótimo \\
\hline 3 & 55 anos & Hemiclônica & $\begin{array}{l}\text { Seqüela de trauma } \\
\text { craniano }\end{array}$ & 1 & ótimo \\
\hline 4 & 2 anos & Braquio-facial & Trauma de parto & 1 & ótimo \\
\hline 5 & 13 anos & $\begin{array}{l}\text { Generalizada tô- } \\
\text { nico-clônica }\end{array}$ & - & 1 & ótimo \\
\hline 6 & 7 anos & Adversiva & Neurocisticercose & 1 & ótimo \\
\hline 7 & 26 anos & $\begin{array}{l}\text { Generalizada tô- } \\
\text { nico-clônica }\end{array}$ & 一 & 1 & ótimo \\
\hline 8 & 14 anos & Hemiclônica & Meningite purulenta & 2 & regular * \\
\hline 9 & 51 anos & Braquio-facial & Tóxica & 1 & ótimo* \\
\hline 10 & 5 meses & Hemiclônica & Meningite purulenta & 2 & ótimo * \\
\hline 11 & 3 anos & Braquio-facial & Trauma de parto & 1 & ótimo \\
\hline 12 & 14 anos & $\begin{array}{l}\text { Ausência mio- } \\
\text { clônica }\end{array}$ & & 1 & ótimo \\
\hline 13 & 4 anos & Braquio-facial & Trauma de parto & 1 & ótimo \\
\hline 14 & 25 anos & $\begin{array}{l}\text { Generalizada tô- } \\
\text { nico-clônica }\end{array}$ & 一 & 1 & ótimo \\
\hline 15 & 12 anos & Adversiva & $\begin{array}{l}\text { Seqüela meningencefa- } \\
\text { lite }\end{array}$ & 2 & rcgular \\
\hline 16 & 28 anos & Hemiclônica & Trauma de parto & 1 & ótimo \\
\hline 17 & 4 anos & Braquio-facial & Seqüela de meningite & 1 & ótimo \\
\hline 18 & 6 anos & Adversiva & Trauma de parto & 1 & ótimo \\
\hline 19 & 3 anos & Hemiclônica & Seqüela de meningitc & 1 & ótimo \\
\hline 20 & 4 meses & Hemiclônica & Meningite purulenta & 2 & $\operatorname{mau} *$ \\
\hline 21 & 6 anos & Hemiclônica & Neurocisticercose & 1 & ótimo \\
\hline 22 & 30 anos & Hemiclônica & Septicemia & 2 & mau * \\
\hline 23 & 3 anos & Braquio-facial & Trauma de parto & 1 & ótimo \\
\hline 24 & 9 anos & $\begin{array}{l}\text { Ausēncia mio- } \\
\text { clônica }\end{array}$ & 一 & 1 & ótino \\
\hline 25 & 4 anos & Hemiclônica & Trauma de parto & 1 & ótimo \\
\hline
\end{tabular}

Tabela 1 - Daclos clinicos dos 25 pacientes em "estado de mal epiléptico" tratados com Valium por via endovenosa. Relaçóes entre a provável etiologia, o tipo de "estado de mal" $e$ o resultado obtido. * Afecsão cerebral aguda.

\section{PESULTADOS}

Nossos resultados são apresentados na tabela 1. Dentre os 20 pacientes com afeção cerebral crônica, 19 tiveram suas crises controladas; em apenas um (caso 15), obtivemos um efeito regular.

No grupo de pacientes com afeccão cerebral aguda (5 casos), dois apresentaram ótimo resultado, sendo que em um dêles (caso 10), foi necessária segunda dose do medicamento. Nos três casos restantes, os resultados foram regulares (caso 8) e maus (casos 20 e 22).

Depressão respiratória ocorreu em apenas um paciente (caso 22). 


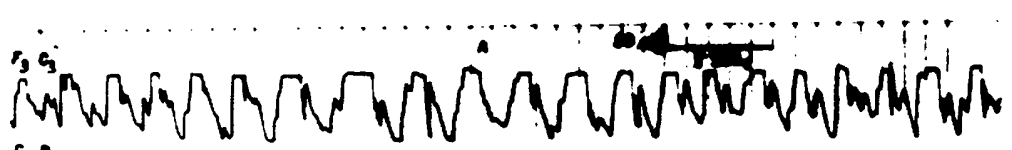

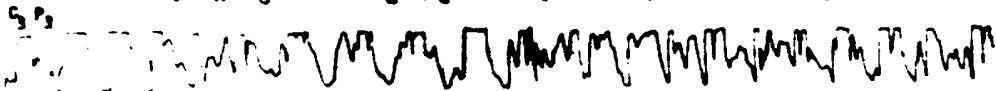

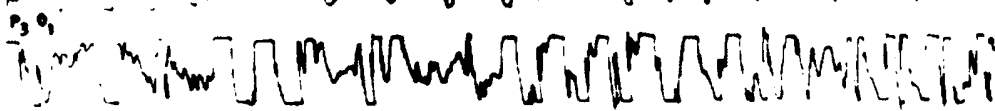
$r_{4} c_{4}$

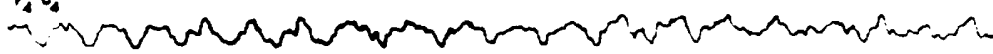

$c_{4} p_{4}$

in 2

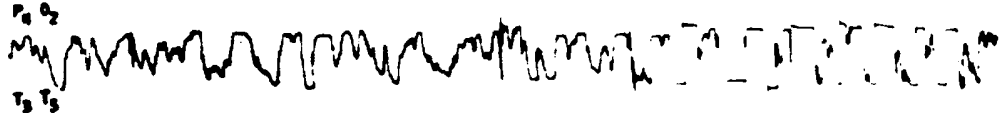

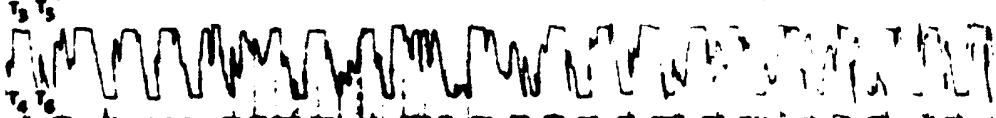

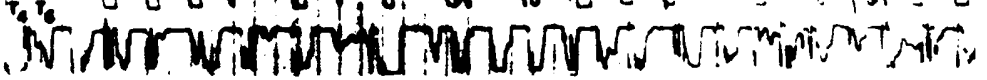
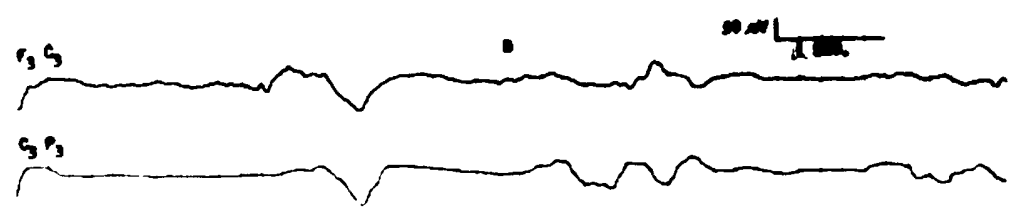

$P_{3} 0_{1}$

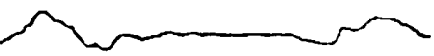

$r_{4} c_{8}$

B

$c_{4} p_{4}$

$P, q_{2}$
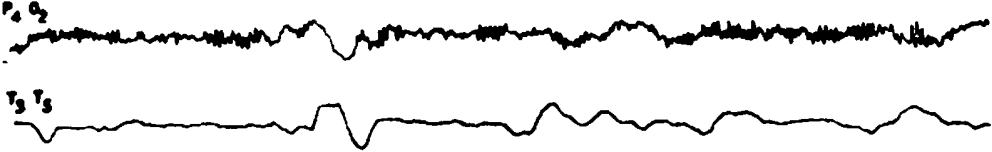

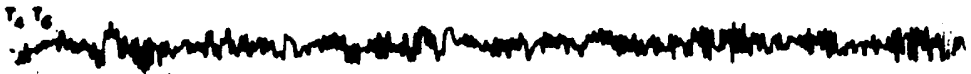

Fig. 1 - Caso 1\%. Estado de mal epiléptico, de tipo parcial (braquiofacial direito). Em A, EEG pré-Valium: atividade paroxistica difusa $e$ contínua predominando no hemisfério cerebral esquerdo. Em $B$, registro pós-Valium (traçado obtido $\$ 0$ segundos após a administração do medicamento): depressão acentuada da atividade de fundo e surtos de ondas lentas de 1 a 2 c/seg., no hemisfério cerebral esquerdo; no hemisfério direito, atividade beta difusa e bem desenvolvida. 


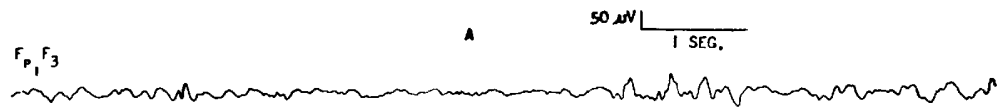

$r_{3} C_{3}$

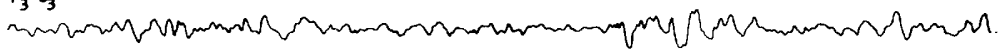

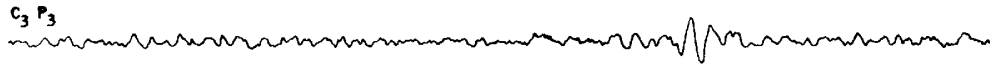

$P_{3} 0_{1}$

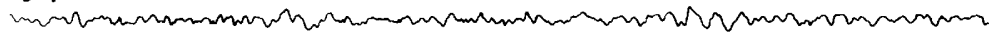
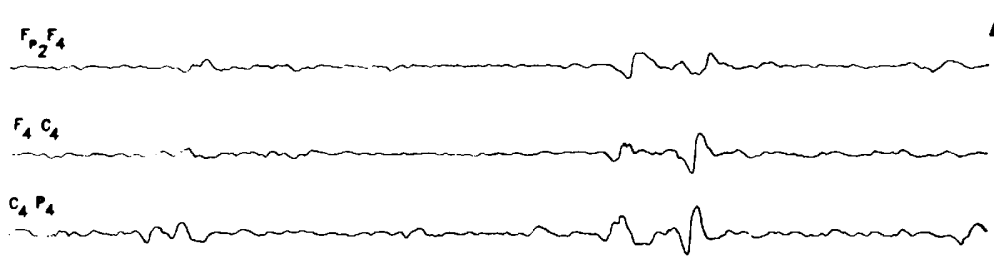

$P_{4} O_{2}$
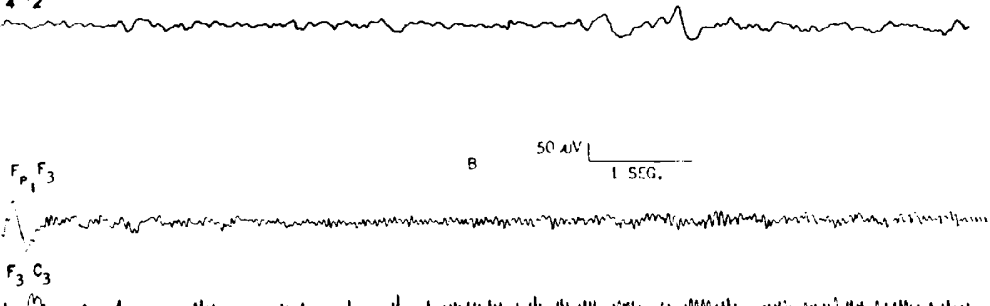

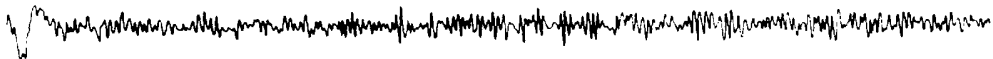
i. N $P_{3}$ $P_{3} \mathbf{O}_{1}$

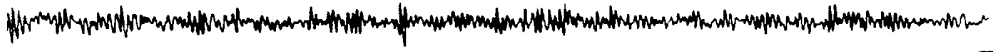
$F_{P_{2}} F_{4}$

$r_{4} \mathrm{C}_{4}$

$c_{4} P_{4}$

$P_{4} a_{2}$

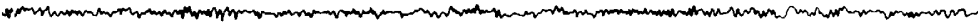

Fig. 2 - Caso 11. Estado de mal epiléptico, de tipo parcial (braquiofacial esquerdo). Em $A, E E G$ pré-Valium: surtos de atividade paroxistica difusa, maior desorganização da atividade de fundo no hemisfério cerebral direito, onde se verifica também depressão do rítmo. Em $B$, registro pós-Valium (traçado obtido 20 segundos após a administração do medicamento): no hemisfério cerebral esquerdo ritmo beta difuso e bem desenvoldo; no direito, depressão da atividade de fundo e ritmo beta menos evidente. 


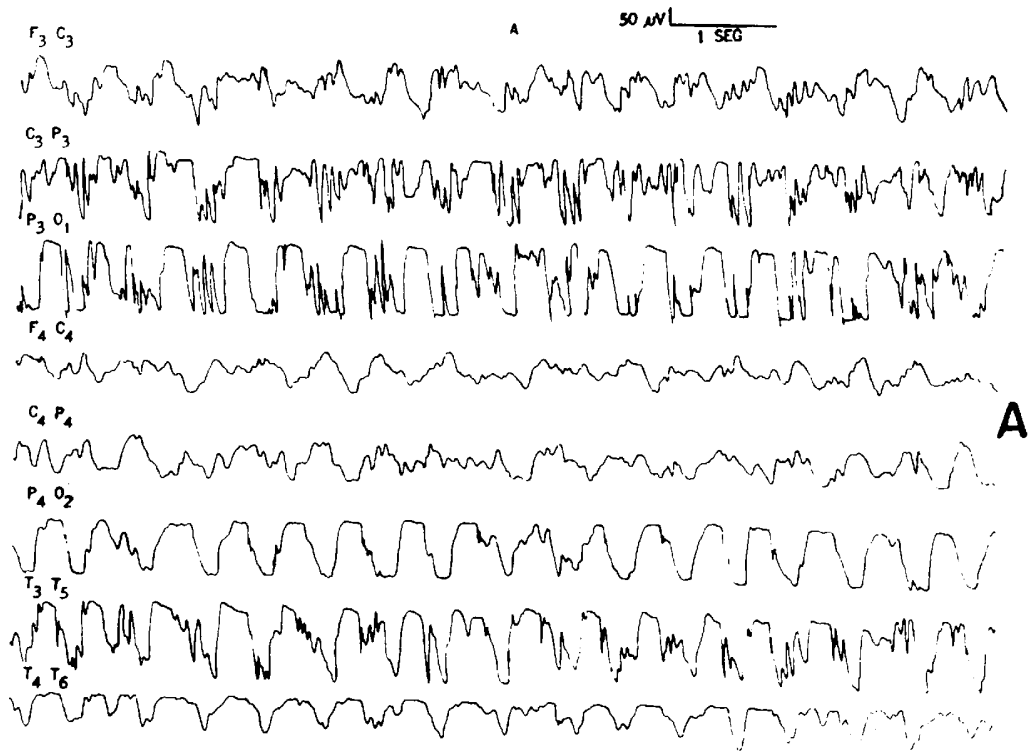

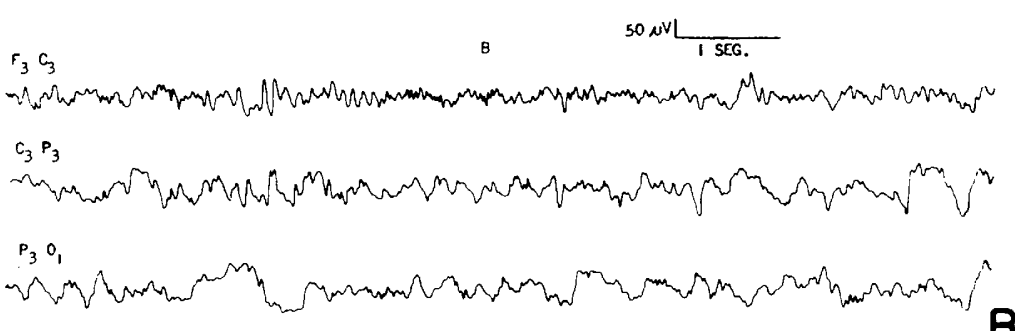

$\mathrm{F}_{4} \mathrm{c}_{4}$

$\mathrm{C}_{4} \mathrm{P}_{4}$

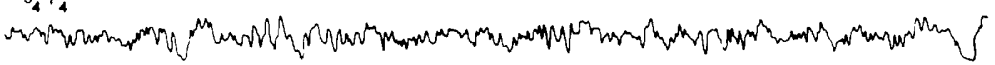

$\mathrm{P}_{4} \mathrm{O}_{2}$

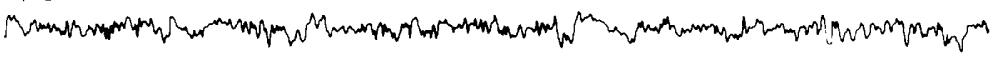

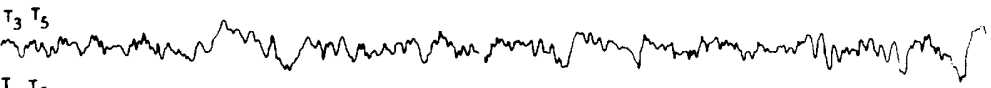

$T_{4} T_{6}$

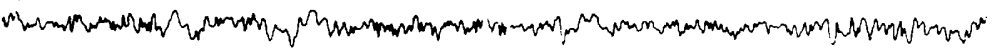

Fig. 3 - Caso 13. Estado de mal epiléptico de tipo parcial (braquiofacial direito). $E m$ A, EEG pré-Valium: atividade paroxistica difusa $e$ continua no hemisfério cerebral esquerdo; no direito, ritmo lento $e$ irregular. Em B, registro pós-Valium (traçado obtido 60 segundos após a administração do medicamento): atividade de fundo lenta e irregular no hemisfério cerebral esquerdo; ritmo mais rápido e melhor organizado no hemisfério cerebral direito. 


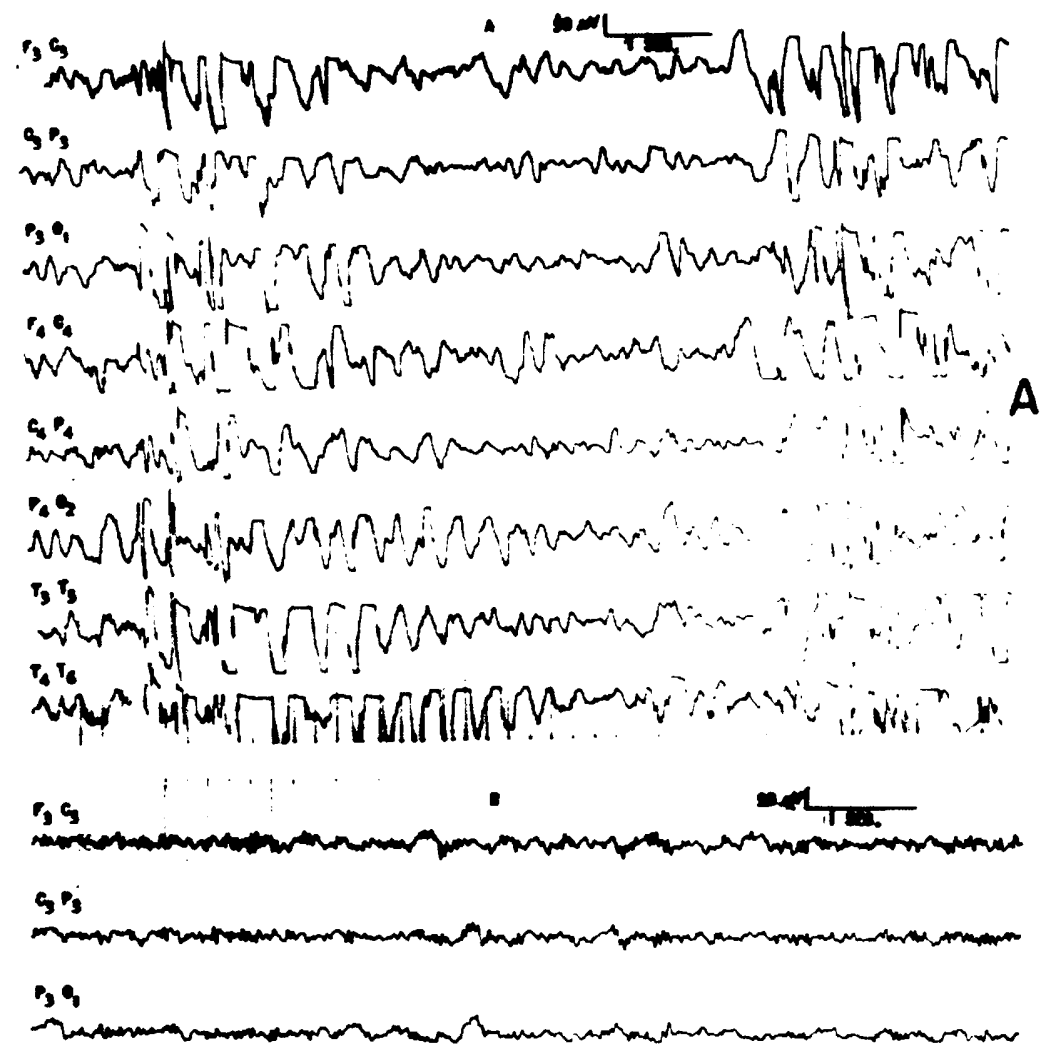

$1, c_{4}$

$c_{4} P_{4}$

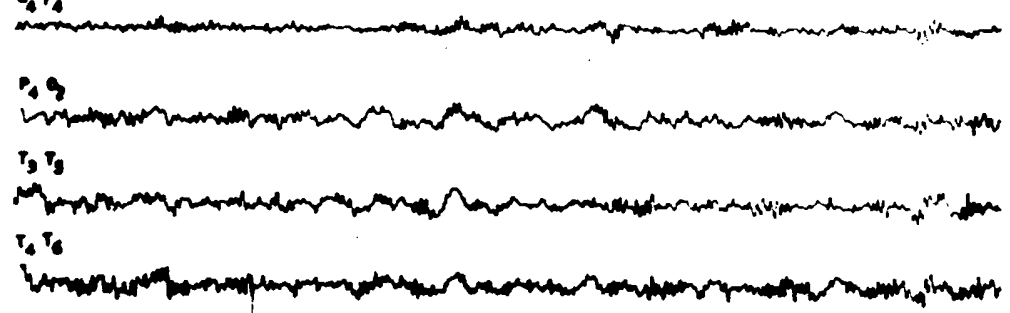

Fig. 4 - Caso 12. Caso de mal epiléptico de tipo generalizado (estado de ausência mioclônica). Em A, EEG pré-Valium: complexos polipontasondas lentas $e$ irregulares de projeção difusa $e$ bilateral. Em $B$, registro pós-Valium (traçado obtido 10 segundos após a administração do medicamento): desaparecimento completo da atividade paroxistica; atividade beta difusa, bilateral e simétrica. 
Em 6 pacientes tivemos a oportunidade de realizar o exame EEG, durante a administração do Valium, e pudemos observar o desaparecimento rápido e completo da atividade paroxística. Cinco dêsses pacientes apresentavam crises lateralizadas e o traçado EEG, imediatamente após a aplicação do Valium, evidenciava depressão acentuada ou desorganização da atividade de fundo, no hemisfério comprometido. No hemisfério normal apareceu uma atividade beta como usualmente ocorre em individuos normais, após a administração de derivados benzodiazepínicos (figs. 1, 2 e 3). O sexto paciente, que apresentava crises de ausência mioclônica, teve seu traçado completamente normalizado, com aparecimento de atividade beta, bilateral e simétrica (fig. 4).

\section{COMENTARIOS}

Valium por via endovenosa tem sido considerado como a droga de escolha no contrôle do "estado de mal epiléptico" 2,8,12. Ao lado de uma ação extremamente eficaz, não produz alterações importantes no estado de consciência, além de raramente determinar alterações no rítmo respiratório $^{2}$ e outros efeitos colaterais indesejáveis, não raras vêzes observados com o uso de barbitúricos por via endovenosa ${ }^{8}$.

Nossos resultados confirmam a eficácia dessa droga no contrôle do "estado de mal". Devemos salientar, entretanto, que as melhores respostas foram obtidas no grupo de pacientes com afecção cerebral crônica (95\%). No grupo de pacientes que exibiam quadro agudo, sòmente dois apresentaram resultados que pcdem ser considerados ótimos. Achados semelhantes foram descritos por Prensky e col. ${ }^{12}$ e Gastaut e col. ${ }^{2}$. Prensky e col. ${ }^{12}$ argumentam, entretanto, que o contrôle do "estado de mal" em pacientes com afecção cerebral aguda é menos efetivo não só com a administração de Valium, mas também com o uso das medicações clássicas.

Com relação aos diferentes tipos de "estados epilépticos", nossos resultados não mostram diferenças importantes, agindo o medicamento eficazmente tanto em crises generalizadas quanto em crises lateralizadas e mioclônicas. Gastaut e col. ${ }^{2}$ relatam bons resultados mesmo no contrôle de "estado de mal" caracterizado por crises tônico-clônicas generalizadas (Grande Mal). Ora, é sabido que o uso per os, por tempo mais ou menos prolongado, dos derivados benzodiazepínicos, particularmente Valium e Mogadon, pode precipitar o aparecimento de crises tipo "Grande Mal", que se tornam mais fàcilmente controláveis ou tendem a desaparecer com a persistência da medicação ${ }^{4,6}$. Por outro lado, temos verificado, no decorrer do tratamento do "Pequeno Mal" pelos derivados benzodiazepínicos, melhora inicial acentuada que tende a desaparecer com o prosseguimento da terapêutica. Resultados semelhantes foram descritos por Gastaut e col. ${ }^{3}$. Essas observações sugerem que o sistema nervoso central frente a essas drogas, reage de maneira diferente em conformidade com a subitaneidade e o tempo de tratamento. Os neurônios comprometidos, sofrendo o "impacto" inicial da droga, apresentariam um estado reacional compativel com um tipo de resposta. Com a persistência da medicação aquêle estado reacional se modificaria por um "mecanismo de hábito" e, conseqüentemente, deixaria de responder. 
Em apenas um paciente, que apresentava quadro septicêmico, encontramos depressão do rítmo respiratório, confirmando a ocorrência dêsse tipo de alteração, na vigência do tratamento do "estado de mal" pelo Valium, em pacientes com patologia cerebral aguda ${ }^{12}$.

Nos casos em que foi possível a realização do EEG durante a administração do Valium, notamos a supressão rápida e completa da atividade paroxística anormal, às vêzes mesmo antes do término da injeção. No hemisfério correspondente ao foco, constatamos depressão acentuada ou desorganização da atividade de fundo e quase ausência de atividade beta, nitidamente presente no hemisfério normal. Achado bastante semelhante foj descrito por Lombroso $^{8}$. Essa assimetria de atividade beta nos parece um elemento importante para a identificação do hemisfério comprometido. No paciente com crises mioclônicas, onde as descargas têm origem em estruturas "centrencefálicas", tal assimetria não foi verificada.

\section{RESUMO E CONCLUSÓES}

Um grupo de 25 pacientes em "estado de mal epiléptico" foi submetido a tratamento pelo Valium endovenoso. Vinte dêsses pacientes apresentavam afecção cerebral crônica e, os restantes, afecção aguda. A droga mostrou-se extremamente eficaz no contrôle das crises, principalmente nos casos com afecção cerebral crônica. Depressão respiratória foi observada em apenas um paciente com afecção cerebral aguda. O eletrencefalograma, obtido durante a administração do Valium, evidenciou o desaparecimento completo da atividade paroxística, que foi substituída, no hemisfério normal, por rítmo beta bem desenvolvido. No hemisfério comprometido o ritmo apresentou-se bastante deprimido ou desorganizado e com discreta ou nenhuma atividade beta.

Baseado em nosso trabalho e naqueles prèviamente relatados, concluimos: 1) Valium endovenoso constitui atualmente a droga de escolha para o contrôle de qualquer tipo de estado de mal epiléptico; 2) em pacientes com afecção cerebral aguda, sua administração deve ser feita com maior cautela em virtude de efeitos colaterais indesejáveis que pode determinar; 3) o exame eletrencefalográfico, obtido durante a administração do Valium, pode ser um elemento importante para se determinar o hemisfério comprometido.

\section{SUMMARY AND CONCLUSIONS}

\section{Intravenous Valium in treatment of status epilepticus}

Twenty five patients in status epilepticus were submitted to treatment by intravenous Valium. Twenty of them were afflicted by chronic cerebral disorders and the others by acute cerebral disorders. The treatment proved to be extremely efficient in the control of the seizures, specially in the cases with chronic lesions. Respiratory depression was observed in only 
one patient afflicted by acute cerebral lesion. Electroencephalograms obtained during the administration of Valium showed complete extinction of the paroxystic activity, which was replaced, in the normal hemisphere, by well developed beta rythm. In the injured hemisphere the background activity was quite depressed or desorganized and presented little or no beta activity.

These findings and those previously reported by others allow the following conclusions: 1) intravenous Valium is presently the drug of choice for the control of any type of status epilepticus; 2) in patients with acute cerebral disorders, the administration of Valium should be made with care, because of its undersirable collateral affects; 3 ) the electroencephalographic examination during the administration of Valium may be important for the determination of which cerebral hemisphere is injured.

\section{REFERENCIAS}

1. GastaUt, H.; CAVENESS, W. F.; LANDOLT, H.; LORENTZ DE HAAS, A. M.; MC NAUGHTON, F. L.; MAGNUS, O.; MERLIS, J. R.; POND, D. A.; RADERMECKER, J. \& STORM VAN LEEUWEN, W. - A proposed international classification of epileptic seizures. Epilepsia 5:297-306, 1964.

2. GASTAUT, H.; ROGER, J.; LOB, H. \& TASSINARI, C. A. - Les nouveaux médicaments anti-épileptiques. L'Encéphale 54:407-421, 1965.

3. GASTAUT, H.; ROGER, J.; SOULAYROL, R.; SAINT-JEAN, M.; TASSINARI, C. A.; REGIS, H.; BERNARD, R.; PINSARD, N. \& DRAVET, C. — L'encéphalopathie épileptique de l'infant avec pointe-ondes lentes diffuses (alias "Petit Mal Variant") ou syndrome de Lennox. Ann. Péd. 42:2093-2103, 1966.

4. GIBBS, F. A. \& ANDERSON, E. M. - Treatment of hypsarhythmia and infantile spasms with a Librium analogue. Neurology 15:1173-1176, 1965.

5. LISKE, E. \& FORSTER, F. M. - Clinical study of a new benzodiazepine as an anticonvulsivant agent. J. New Drugs 3:241-244, 1963.

6. LISON, M. P. \& MEGA, D. - Espasmos em flexão: estudo clínico e eletrencefalográfico de paciente tratados com Ro 4-5360. Arq. Neuro-Psiquiat. (São Paulo) 25:87-105, 1967.

7. LIVINGSTON, S. - The epilepsies: diagnosis and treatment. In Disease-amonth, Year Book Medical Publishers, Chicago, 1967.

8. Lombroso, C. T. - Treatment of status epilepticus with Diazepam. Neurology 16:629-634, 1966.

9. MARKHAM, C. H, - The treatment of myoclonic seizures of infancy and childhood with LA-1. Pediatrics 34:511-518, 1964.

10. MILlichaP, J. G. \& ORTIZ, W. R. - Nitrazepam in myoclonic epilepsies. Am. J. Dis. Child. 112:242-248, 1966.

11. MULleR, H. R.; KLINGLER, M.; KAESER, H. E.; WURMSER, P. \& HIRT, H. R. - Zur Behandlung des Status Epilepticus mit Diazepam (Valium). Erfahrungen in 12 Fällen. Schweiz. med. Wschr. 96:121-127, 1966. 
154 ARQ. NEURO-PSIQUIAT. (SÄO PAULO) VOL. 26, N.० 2, JUNHO, 1968

12. PRENSKY, A. L.; RAFF, M. C.; MOORE, M. J. \& SCHWAB, R. S. - Intravenous Diazepam in treatment of prolonged seizure activity New Eng. J. Med. 276:779-784, 1967.

13. RALLO PIQUE, E. \& HENKING, R. - Expérimentation clinique et électroencéphalographique du Diazepam intraveineux chez les malades épileptiques. Psychiat. Neurol, 150:214-229, 1965. Brasil.

Departamento de Neurologia - Faculdade de Medicina - Ribeirão Prêto, $S P$ - 